\title{
Ranolazine for the symptomatic treatment of patients with chronic angina pectoris in Greece: a cost-utility study
}

\author{
Georgia Kourlaba ${ }^{1 *}$, Charalambos Vlachopoulos ${ }^{2}$, John Parissis ${ }^{3}$, John Kanakakis ${ }^{4}$, George Gourzoulidis ${ }^{5}$ \\ and Nikos Maniadakis ${ }^{5}$
}

\begin{abstract}
Background: To conduct an economic evaluation comparing ranolazine as add-on therapy to standard-of-care (SOC) with SoC alone in patients with stable angina who did not respond adequately to first line therapy, in Greece.

Methods: A decision tree model was locally adapted in the Greek setting to evaluate the cost-utility of ranolazine during a 6-month period. The analysis was conducted from a third-party payer perspective. The clinical inputs were extracted from the published literature. The cost inputs considered in the model reflect drug acquisition, hospitalizations, vascular interventions and monitoring of patients. The resource utilization data were obtained from 3 local experts. All costs refer to the year 2014. Cost-effectiveness was assessed by means of the incremental cost per quality adjusted life year (QALY) gained with the ranolazine as add-on therapy relative to SoC alone (ICER). Probabilistic sensitivity analysis (PSA) was performed.
\end{abstract}

Results: Ranolazine as add-on therapy was more costly compared to SoC alone, as the 6-month total cost per patient was $€ 1170$ and $€ 984$, respectively. Patients received ranolazine plus SoC and SoC alone gained 0.3155 QALYs and 0.2752 QALYS, respectively. Ranolazine plus SoC resulted in an ICER equal to $€ 4620$ per QALY gained, well below the threshold of $€ 34,000$ per QALY gained. The PSA showed that the likelihood of ranolazine plus SoC being cost-effective at the threshold of $€ 34,000$ per QALY gained was $100 \%$.

Conclusions: The results suggest that ranolazine as add-on treatment may be a cost-effective alternative for the symptomatic treatment of patients with chronic stable angina in Greece.

\section{Background}

Chronic stable angina is one of the most common conditions experienced by patients with heart disease with significant detrimental effects on health related quality of life, including pain, poor general health status, psychological distress and restriction of activity $[1,2]$. Moreover, there is evidence that patients with moderate or severe angina have more than a twofold higher risk for mortality compared to those with minimal or mild angina [3]. Apart from mortality and morbidity, chronic stable angina may impose great economic burden for

\footnotetext{
* Correspondence: kurlaba@gmail.com

${ }^{1}$ The Stavros Niarchos Foundation-Collaborative Center for Clinical Epidemiology and Outcomes Research (CLEO), National and Kapodistrian University of Athens, School of Medicine, Athens, Greece

Full list of author information is available at the end of the article
}

payers, health care systems [4], and patients as this condition requires long-term pharmacological treatment, it leads to frequent hospital admissions, requiring in many cases expensive vascular interventions. Hospitalizations, both with and without revascularization procedures are the main cost drivers as they account for almost two thirds of the total health care expenditures related to chronic stable angina [4-6]. In addition to its direct medical costs, chronic angina has been found to lead to substantial productivity loss [7]. To be more precise, studies have shown that the total cost (both direct and indirect) of chronic angina is 2 to 3 times higher compared to the direct one alone [7].

In this context, it is clear that the effective management of chronic stable angina is important both for 
clinical and economic reasons. Effective treatment of chronic stable angina should aim at reducing or ideally abolishing symptoms, improve quality of life and prognosis [2]. Lifestyle changes, pharmacological therapy and revascularization procedures all have important roles in the management of stable angina [8-10]. The pharmacological management of stable angina includes, nitrates, b-blockers, calcium antagonists, statins, etc. [8-10]. Nonetheless, despite the aggressive use of conventional antianginal therapies, many patients experience persistent angina $[11,12]$. For this reason, the need for additional antianginal agents with novel mechanisms of action arose. Several new drug have been tested for the management of chronic stable angina [13-16]. Ranolazine (Ranexa ${ }^{\circ}$ ) is one such antianginal agent [12]. Ranolazine is a drug that reduces angina symptoms, with a mechanism of action different from that of currently available pharmacological therapies [17-20]. It was approved on July 9, 2008 by the European Medicines Agency for use in patients with chronic angina, who continue to be symptomatic on bblockers, and/or calcium antagonists [21].

The efficacy and safety of ranolazine as add-on treatment for stable angina has been evaluated in three clinical trials (MARISA [14], CARISA [17] and ERICA [22]) and one large trial of patients with non-ST elevation acute coronary syndrome (MerlinTIMI 36 [23]). These clinical trials showed an improvement in exercise performance and a decrease in angina attacks.

Although, it is proven that ranolazine is an effective treatment option for the management of chronic stable angina, it may also impose a tangible cost to the health care system and payers. Greece is going through a significant economic crisis, which has resulted in major budget constraints on the national healthcare system. Under these circumstances, there is an increasing need for using therapeutic options which are not only clinically effective but also economically efficient, in order to maximize the value for money attained from the scarce resources invested in health care. In this context, the aim of the present study was to evaluate the cost-utility of ranolazine as add-on therapy to standard-of-care (SoC) compared to SoC alone in patients with stable angina who did not respond adequately to first line therapy with b-blockers and/or calcium channel antagonists in the health care setting of Greece.

\section{Methods}

A decision tree model [24] was locally adapted to evaluate the cost-utility of ranolazine as add-on therapy to SoC, compared to SoC alone, during a 6-month period (time horizon) in patients with stable angina, who did not respond adequately to first line therapy with b-blockers and/or calcium channel antagonists in
Greece. The analysis was conducted from a third-party payer perspective (National Organization for Healthcare Services Provision [EOPYY]). Because the time horizon did not exceed 1 year, no discounting was performed both to cost and health outcomes. The model led to calculation of incremental cost incurred and incremental quality adjusted life years (QALYs) gained using ranolazine as add-on treatment for stable angina. The cost-effectiveness of ranolazine was expressed as an incremental cost-effectiveness ratio (ICER) relative to SoC. As this study is an economic evaluation analysis and does not involve human subjects no ethics approval issues arise. Input data including human material or human data were derived from other published studies performed with the approval of an appropriate ethics committee [22, 23, 25-27].

\section{Model structure}

The model used is a decision tree constructed on Microsoft Excel (Fig. 1), which has two main cohorts of patients representing both treatment alternatives. On the one hand, the ranolazine branch represents the treatment using ranolazine as an add-on therapy for the symptomatic treatment of angina pectoris. On the other hand, the "base treatment" branch represents the standard treatment in the same type of patients. The ranolazine branch is divided into two sub-branches, which shows the possibility of adherence or non-adherence by the patients to the treatment. Patients who do not adhere to the treatment with ranolazine are treated immediately with standard treatment, however for the purpose of the model and following the principle of intention to treat, these patients remain in the ranolazine branch. Whether or not the patient adheres to the treatment, four possible scenarios are considered, related to the frequency of angina that might be experienced at this stage of the model: minimal, mild, moderate or severe frequency. The transition probabilities depend on whether the patient has continued the treatment with ranolazine, whether the treatment has been stopped by the patient, or whether the patient belongs to the cohort of patients with standard treatment. Once the patient is in one of these potential angina frequency groups, hospitalization may or may not be required. When hospitalization is required, then an intervention might be needed or not. Most frequently required interventions at this stage are coronary artery bypass graft (CABG), or percutaneous coronary intervention (PCI). We did not consider mortality in this model related to stable angina pectoris, because, it has been shown in different studies that there are not significant differences in the mortality of patients under evaluation [23, 28]. 


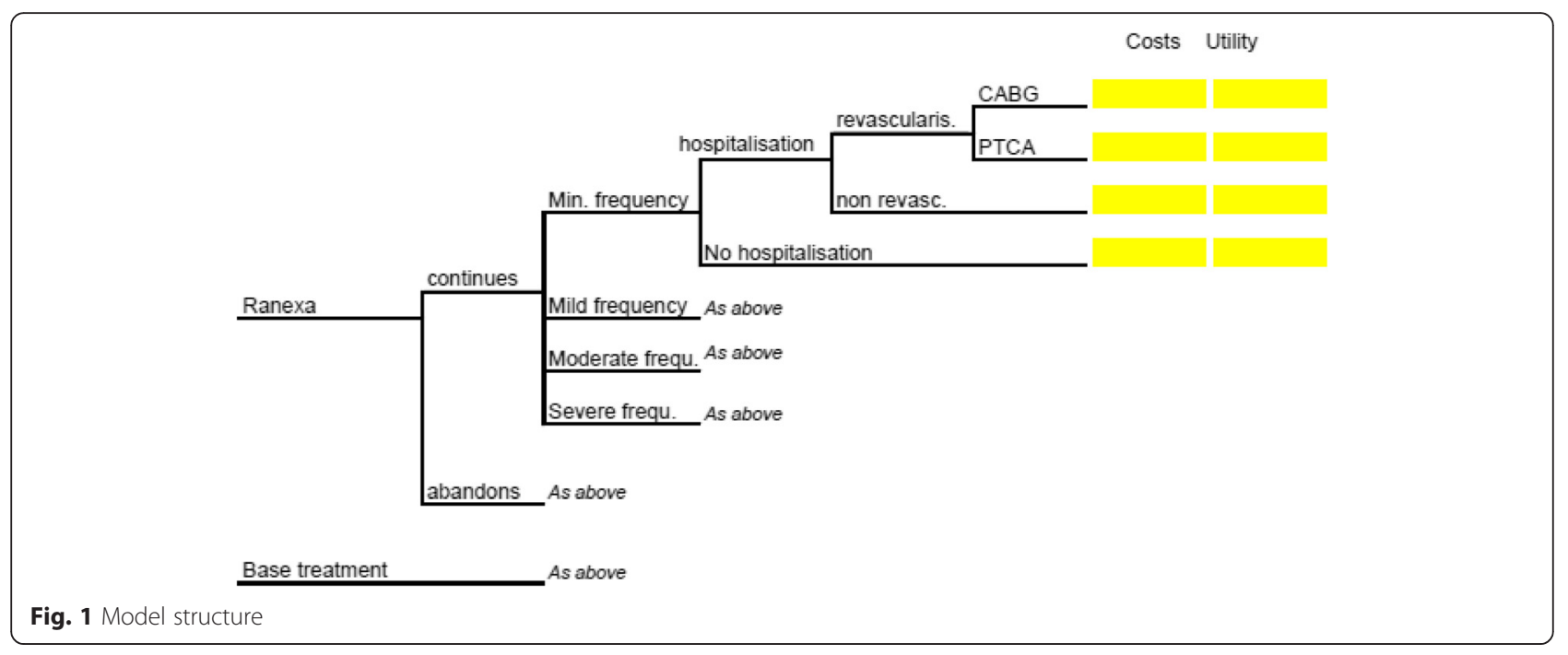

\section{Clinical inputs}

The clinical inputs considered in the model were: a) transition probabilities, including the probability of experiencing minimum, mild, moderate or severe angina frequency, the probability of being hospitalized, and the probability of being subjected to a revascularization procedure, and b) utility values related to non-hospitalization, hospitalization without revascularization, and hospitalization with revascularization. The above clinical inputs considered in the present analysis were obtained from relevant clinical and epidemiological trials [22, 23, 25-27]. It is worth noting that the same published studies were used in a previous publication of the model [24].

\section{Transition probabilities}

As mentioned above, the transition probabilities considered in the present model are presented in detail elsewhere [24]. In brief, the probability of adherence to ranolazine treatment was estimated at $72 \%$ [23] while the odds of experiencing at least a minimal frequency of angina (out of minimal, mild, moderate or severe) episode was estimated according to the treatment received [22] (Table 1). At this point it should be noticed that the frequency of angina classification depended on the selfadministered questionnaire answers. The questionnaire used was the Seattle angina questionnaire [29]. It led to grouping of patients onto different categories according to their responses. To be more precise, patients with $<0.5$ episodes/week, 0.5 -3 episodes/week, 3-5.5 episodes/week and $>=5.5$ episodes/week were classified to experience minimal, mild, moderate and severe angina, respectively.

Moreover, the 6-month probability of hospitalization have been estimated at 20,20,27 and $30 \%$ for minimal, mild, moderate or severe angina frequencies respectively, based on data obtained from Merlin-TIMI 36 clinical trial [25] (Table 1).

Of note, it has been found that the probability of hospitalization is independent of the type of treatment (ranolazine or SoC) but it depends on the severity of angina. When hospitalization is required, an intervention might be needed (or not) such as CABG or PCI/Stent [26] (Table 1). All these data were reviewed and validated by 3 local experts (C.V., J.P., J.K.) in order to increase the credibility and validity of the model inputs.

\section{Utility weights}

QALYs were selected as the measure of effectiveness in present study. Utility weights are a measure of a patient's preference of a particular health state and generally range from 0 (death) to 1 (perfect health). Utility values were obtained from the literature [27] (Table 1).

\section{Costing methodology}

Since the analysis was conducted from the public thirdparty-payer perspective (EOPYY), only health care costs reimbursed by the payer were included in the model. The total reimbursement cost reflected and encapsulated all the possible healthcare resource consumption of patients during the horizon of analysis (6 months). The average resource utilization for a Greek patient suffering from stable angina was extracted from 3 the local experts using a questionnaire developed to serve the purpose of the present study. The questionnaire was developed to reflect the disease management in Greece, and the local experts participating in the present study confirmed its content validity. In this context, the cost of anti-anginal drug acquisition, hospitalizations, vascular interventions and monitoring tests including outpatient visits, laboratory tests and imaging diagnostic 
Table 1 Clinical inputs considered in the model

\begin{tabular}{|c|c|c|}
\hline & \multicolumn{2}{|c|}{ Value of the parameter } \\
\hline & Ranolazine (\%) & SoC (\%) \\
\hline \multicolumn{3}{|l|}{ Transition probabilities } \\
\hline Adherence to treatment with ranolazine [23] & 0.720 & \\
\hline \multicolumn{3}{|l|}{ Angina frequency [22] } \\
\hline Minimum & 0.040 & 0.000 \\
\hline Mild & 0.605 & 0.071 \\
\hline Moderate & 0.208 & 0.689 \\
\hline Severe & 0.137 & 0.241 \\
\hline \multicolumn{3}{|l|}{ Hospitalization [25] } \\
\hline With minimum angina frequency & 0.200 & 0.200 \\
\hline With mild angina frequency & 0.200 & 0.200 \\
\hline With moderate angina frequency & 0.270 & 0.270 \\
\hline With severe angina frequency & 0.300 & 0.300 \\
\hline \multicolumn{3}{|l|}{ Revascularization [26] } \\
\hline Revascularization & 0.500 & 0.500 \\
\hline Revascularization with CABG & 0.280 & 0.280 \\
\hline Revascularization with PTCA & 0.720 & 0.720 \\
\hline \multicolumn{3}{|l|}{ Utility values [27] } \\
\hline No hospitalization, minimum angina frequency & 0.810 & 0.840 \\
\hline No hospitalization, mild angina frequency & 0.750 & 0.750 \\
\hline No hospitalization, moderate angina frequency & 0.600 & 0.600 \\
\hline No hospitalization, severe angina frequency & 0.390 & 0.390 \\
\hline Hospitalization without revascularization, minimum angina frequency & 0.800 & 0.800 \\
\hline Hospitalization without revascularization, mild angina frequency & 0.740 & 0.740 \\
\hline Hospitalization without revascularization, moderate angina frequency & 0.590 & 0.590 \\
\hline Hospitalization without revascularization, severe angina frequency & 0.380 & 0.380 \\
\hline Revascularization, minimum angina frequency & 0.750 & 0.750 \\
\hline Revascularization, mild angina frequency & 0.690 & 0.690 \\
\hline Revascularization, moderate angina frequency & 0.540 & 0.540 \\
\hline Revascularization, severe angina frequency & 0.330 & 0.330 \\
\hline
\end{tabular}

CABG: coronary artery bypass graft, PCI/STENT: percutaneous transluminal angioplasty \& stenting, SoC: standard of care

examinations were considered in the analysis. All costs reflect the year 2014 $(€)$.

\section{Drug acquisition cost of anti-anginal drugs}

Drug acquisition costs of ranolazine and SoC were calculated using the latest price bulletin issued by the Ministry of Health (26.11.2014) [30], as well as the corresponding reimbursement prices (Positive List for the reimbursement of medicines, FEK 3376/16.12.14). The reimbursement prices were reduced by the patient relevant patient co-payment $(25 \%)$ and relative rebates as they suggested by the corresponding legislation (Official Government Gazzete, FEK 64/16.1.2014). In particular, the rebate of $9 \%$ given by manufacturers to get into the positive list was considered in the analysis. For ranolazine only, an additional rebate of $2 \%$ was considered as it is alone in its cluster (Official Government Gazzete, FEK 64/16.1.2014). At this point, it should be pointed out that the volume-related rebate ranging from 2 to $12 \%$ could have been considered in the analysis. Due to lack of volume -related data for all drugs, it was not taken into account in the base case analysis.

Standard care drug costs have been modelled using the overall proportions of patients using each therapeutic class and the mean daily dose for each drug as obtained from the 3 local experts and the relevant drug acquisition cost, calculated as mentioned above. For each therapeutic class, the active substances (INN) considered in the analysis reflected the most commonly prescribed drug locally, as obtained from the 3 local 
experts. Hence, a weighted cost was calculated for each therapeutic class based on the distribution of patients to different active substances. Based on those mentioned above, the total 6-month acquisition cost for SoC was calculated at $159.01 €$.

Regarding ranolazine, based on data provided by local experts it was considered that during the first two months $80 \%$ of patients received $750 \mathrm{mg}$ (375 mg twice daily) as a daily dose, while the remaining $20 \%$ received $1000 \mathrm{mg}$ (500 mg twice daily) as a daily dose and from the third month and onwards $50 \%$ of patients received $750 \mathrm{mg}$ (375 mg twice daily) as a daily dose, while $40 \%$ of patients received $1000 \mathrm{mg}$ (500 mg twice daily) as a daily dose and the remaining $10 \%$ received $1500 \mathrm{mg}$ (750 mg twice daily) per day. The total 2-month and 4-month acquisition cost for ranolazine was calculated at $89.27 €$ and $178.75 €$ respectively (Additional file 1 : Table S1).

\section{Hospitalization costs}

To estimate the total 6-month hospitalization cost (excluding hospitalizations related to vascular interventions), the reimbursement tariff per hospitalization obtained from the Diagnostic Related Groups (DRGs) tariffs' list issued by the Greek Ministry of Health [31] was multiplied by the number of hospitalizations, as provided by the 3 local experts. The hospitalization cost at a 6-month period was found to range from $€ 226.67$ in patients experiencing minimal angina episodes to $€ 2451$ in patients experiencing severe angina episodes [Table 2].

Regarding the cost of vascular interventions, the proportion of patients with stable chronic angina undergoing revascularization (i.e. PCI or CABG), as extracted from the literature [26] and validated by the 3 local experts, was combined with the reimbursed tariff per revascularization obtained from the relevant DRGs tariffs' lists issued by the Greek Ministry of Health [31]. The cost per CABG and $\mathrm{PCI} /$ stent were found to be $€ 5772$ and $€ 1798$ respectively. To account for the effect of angina severity to the hospitalization cost, the model allowed the proportion of patients requiring hospitalization (with and without revascularization) and the hospitalization frequency to depend on angina severity.

\section{Routine monitoring costs}

Routine monitoring costs include outpatient visits, laboratory tests (e.g. blood and biochemical tests) and diagnostic tests. The number of visits, laboratory tests and diagnostic tests (e.g. echo, MRI etc.) required as well as the proportion of patients undergoing each test were retrieved from the 3 local experts. The corresponding reimbursed unit costs were obtained from the Government Gazette (FEK $\left.A^{\prime} 262 / 16-12-2011\right)$ and from the official site of EOPYY respectively.

The 6-month cost for outpatient visits was found to be extremely low ranging from $€ 7$ in patients suffering from minimal angina episodes to $€ 31.33$ in those suffering from severe angina. With respect to laboratory and diagnostic tests, it was found that the 6-month laboratory test cost varies from $€ 7.93$ to $€ 40.29$ and the 6-month diagnostic tests cost from $€ 40.72$ to $€ 123.79$ [Table 2].

As with hospitalizations, the model allowed the proportion of patients undergoing to laboratory and diagnostic tests, as well as the number of tests and visits to depend on angina severity.

\section{Data analysis}

The aforementioned data were used to get mean estimates of QALY, and total direct costs related to each comparator (ranolazine $+\mathrm{SoC}$ vs. SoC alone). The costeffectiveness of ranolazine plus $\mathrm{SoC}$ over $\mathrm{SoC}$ alone was evaluated by calculating the incremental cost per QALY gained (ICER). For a treatment to be considered costeffective a willingness-to-pay (WTP) threshold of $€ 34,000$ per QALY was used in the current analysis. This is based on the WHO guidelines stating that a treatment should be considered cost-effective if the ICER is up to 3 times the GDP per capita of that country and a treatment is considered highly cost effective at less than 1 times the GDP per capita [32]. The GDP per capita in Greece was estimated at $€ 17,000$ taken from the IMF estimation of GDP per capita using current prices [33].

Table 2 Other medical costs considered in the model

\begin{tabular}{lcccr}
\hline Medical cost for 6-month period & & & \\
\hline Angina frequency & Hospitalization cost $(€)^{\mathrm{a}}$ & Outpatient visits cost $(€)^{\mathrm{b}}$ & ${\text { Cost of laboratory tests }(€)^{c}}^{\text {C Cost of diagnostic tests }(€)^{c}}$ \\
\hline Minimal & 226.67 & 7 & 7.93 & 40.72 \\
Mild & 453.33 & 9.17 & 9.41 & 64.55 \\
Moderate & 566.67 & 19.33 & 21.78 & 97.02 \\
Severe & 2450.83 & 31.33 & 40.29 & 123.79 \\
\hline
\end{tabular}

${ }^{\mathrm{a}}$ Official source: Based in local expert's opinion and DRG such as K36X (€ 340) and K36M (€ 865) , DRG cost were obtained from FEK 9468/27-3-12

b Official source: This cost was obtained by combining of the resources (average of number of visits and \% of patients), as they provided by the local expert's opinion with the corresponding unit costs obtained from government gazette (FEK A'262/16-12-2011)

'Official source: The cost assigned was calculated based on resources used such as average of number of tests and \% of patients). These resources were obtained from the local expert's opinion and these resources were combined with the related unit costs obtained from official site of EOPYY 
The majority of input data used in the current model are subjected to variation. Therefore, in order to deal with uncertainty, a probabilistic sensitivity analysis (PSA) was performed using a second-order Monte Carlo simulation. In this analysis, a distribution was assigned around each parameter (i.e. costs, transition probabilities etc.) and the aforementioned economic and health outcomes associated with simultaneously selecting random values from those distributions were generated. Distributions were selected based on the nature of variables [34]. In particular, a gamma distribution was used to represent the uncertainty in costs, because costs are constrained on the interval zero to positive infinity and are often highly skewed. Binomial parameters (i.e. the probability of hospitalization) and utility values are constricted on the interval zero to one and hence they were varied according to beta distribution. For multinomial data such as the probability of experiencing a minimal, mild, moderate or severe angina episode, a Dirichlet distribution was used. Then, 5000 estimates of costs, LY, QALYs, and incremental cost per QALYs were obtained by applying the bootstrapping technique. The mean values of the bootstrapped estimates represent unbiased estimations of the parameters under investigation. The bootstrap percentile method was used to obtain the uncertainty appropriate intervals for each parameter [35]. A cost-effectiveness acceptability curve (CEAC) was plotted, showing the proportion of simulations that are considered cost-effective at different levels of WTP per QALY gained.

Moreover, a one-way sensitivity analysis (OWSA) was undertaken to test the robustness of the results, by varying individual parameters between low and high values, in order to ascertain the key drivers of costeffectiveness. The upper and lower bounds of all parameters were set at $\pm 20 \%$ (assumption) of the base case values. The parameters evaluated in this analysis were the cost of ranolazine, the cost of SoC, the cost of hospitalization with no revascularization, the cost of hospitalization with CABG, the cost of hospitalization with $\mathrm{PCI} /$ Stent, the probability of patients continuing on ranolazine therapy, the probability of hospitalization with minimal angina, the probabilities of hospitalization, and the utility values. Additionally, one scenario was also considered for the time horizon of the model, assuming that it was 1 year instead of 6 months used in the base case analysis.

All statistical calculations performed using Microsoft Excel 2010.

\section{Results \\ Deterministic results}

The analysis showed that, the total 6-month cost per patient was $€ 1170$ and $€ 984$, for ranolazine plus SoC and SoC alone. In terms of health outcomes, the analysis revealed that ranolazine was more effective compared to SoC alone in terms of QALYs. Patients who received ranolazine plus SoC gained 0.3155 QALYs while patients who received SoC alone gained 0.2752 QALYs. Under the base case assumptions, incremental analysis showed that ranolazine plus SoC resulted in an incremental cost of $€ 4620$ per QALY gained, well below the predetermined WTP threshold of $€ 34,000$ per QALY gained [Table 3].

\section{One-way sensitivity analysis}

The OWSA revealed that the results of the model were more sensitive to the utility for no hospitalization of mild angina severity as this parameter was found to have the greatest effect on ICER followed by the probability of patients continuing on ranolazine therapy and the utility for no hospitalization of moderate angina severity. It is worth noting that in all variations in the parameters which were considered in the sensitivity analysis the ranolazine remains a cost-effective treatment, since the ICER per QALY gained remains well below of the threshold of $€ 34,000$ per QALY gained.

Moreover, when the scenario of 1 year time horizon was considered in the analysis, ranolazine remained a cost-effective treatment resulting in an ICER equal to $€ 6579$ per QALY gained (data not presented in details) [Table 4].

Table 3 Deterministic \& probabilistic results for ranolazine plus SoC vs SoC alone

\begin{tabular}{|c|c|c|c|c|c|c|}
\hline \multirow{3}{*}{ Costs } & \multicolumn{3}{|c|}{ Deterministic results } & \multicolumn{3}{|l|}{ Probabilistic results } \\
\hline & \multirow[t]{2}{*}{ Ranolazine } & \multirow[t]{2}{*}{ SoC } & \multirow[t]{2}{*}{ Incremental } & Ranolazine & \multirow[t]{2}{*}{ SoC } & \multirow[t]{2}{*}{ Incremental } \\
\hline & & & & Mean $(95 \% \mathrm{Cl})$ & & \\
\hline Total Costs $(€)$ & 1170 & 984 & 186 & $1170(1123 ; 1213)$ & $985(933 ; 1036)$ & $184(154 ; 216)$ \\
\hline \multicolumn{7}{|c|}{ Health outcomes } \\
\hline QALYS & 0.3155 & 0.2752 & 0.0403 & $0.3157(0.3093 ; 0.3221)$ & $0.2754(0.2672 ; 0.2836)$ & $0.0402(0.0345 ; 0.0456)$ \\
\hline \multicolumn{7}{|c|}{ Incremental analysis } \\
\hline ICER per QAL & & & 4620 & & & $4904(3526 ; 5962)$ \\
\hline
\end{tabular}

ICER: incremental cost effectiveness ratio, QALY: quality-adjusted life year, SoC: standard of care, Cl: confidence interval 
Table 4 Results of one-way sensitivity analysis

\begin{tabular}{|c|c|c|c|c|c|}
\hline Input parameter & Base case value & aLow value & ICER & aHigh value & ICER \\
\hline Cost of ranolazine & $€ 268$ & $€ 214$ & $€ 3511$ & $€ 322$ & $€ 5725$ \\
\hline Cost of SoC & $€ 159$ & $€ 127$ & $€ 4598$ & $€ 191$ & $€ 4643$ \\
\hline Cost of hospitalization with no revas (mid, minimal, moderate) & $€ 340$ & $€ 272$ & $€ 4692$ & $€ 408$ & $€ 4549$ \\
\hline Cost of hospitalization with no revas (severe) & $€ 865$ & $€ 692$ & $€ 4438$ & $€ 1038$ & $€ 4803$ \\
\hline Cost of hospitalization with CABG & $€ 5772$ & $€ 4617$ & $€ 4841$ & $€ 6926$ & $€ 4611$ \\
\hline Cost of hospitalization with $\mathrm{PCI} /$ Stent & $€ 1797$ & $€ 1438$ & $€ 4712$ & $€ 2157$ & $€ 4528$ \\
\hline Probability of patients continuing on Ranolazine therapy & 0.72 & 0.576 & $€ 7539$ & 0.864 & $€ 2675$ \\
\hline Probability of hospitalization with minimal angina & 0.20 & 0.16 & $€ 4570$ & 0.24 & $€ 4671$ \\
\hline Probability of hospitalization with mild angina & 0.20 & 0.16 & $€ 3858$ & 0.24 & $€ 5393$ \\
\hline Probability of hospitalization with moderate angina & 0.27 & 0.21 & $€ 5572$ & 0.31 & $€ 3684$ \\
\hline Probability of hospitalization with severe angina & 0.30 & 0.24 & $€ 5497$ & 0.36 & $€ 3747$ \\
\hline Utility for no hospitalization (minimal angina) & 0.81 & 0.65 & $€ 4842$ & 0.97 & $€ 4418$ \\
\hline Utility for no hospitalization (mild angina) & 0.75 & 0.60 & $€ 10,076$ & 0.90 & $€ 2938$ \\
\hline Utility for no hospitalization (moderate angina) & 0.60 & 0.48 & $€ 3369$ & 0.72 & $€ 7349$ \\
\hline Utility for no hospitalization (severe angina) & 0.39 & 0.31 & $€ 4391$ & 0.47 & $€ 4875$ \\
\hline Utility for hospitalization without revas (minimal angina) & 0.80 & 0.64 & $€ 4647$ & 0.96 & $€ 4594$ \\
\hline Utility for hospitalization without revas (mild angina) & 0.74 & 0.59 & $€ 4976$ & 0.89 & $€ 4312$ \\
\hline Utility for hospitalization without revas (moderate angina) & 0.59 & 0.47 & $€ 4335$ & 0.71 & $€ 4946$ \\
\hline Utility for hospitalization without revas (severe angina) & 0.38 & 0.30 & $€ 4572$ & 0.46 & $€ 4670$ \\
\hline Utility for hospitalization with revas (minimal angina ) & 0.75 & 0.60 & $€ 4645$ & 0.90 & $€ 4596$ \\
\hline Utility for hospitalization with revas (mild angina) & 0.69 & 0.55 & $€ 4955$ & 0.83 & $€ 4328$ \\
\hline Utility for hospitalization with revas (moderate angina) & 0.54 & 0.43 & $€ 4353$ & 0.65 & $€ 4922$ \\
\hline Utility for hospitalization with revas (severe angina) & 0.33 & 0.26 & $€ 4576$ & 0.40 & $€ 4666$ \\
\hline
\end{tabular}

SoC: standard of care, CABG: coronary artery bypass graft, PCI/STENT: percutaneous transluminal angioplasty\& stenting, Revas: revascularization

a Low and high values currently based on $\pm 20 \%$ variation from the base case

\section{Probabilistic sensitivity analysis}

The PSA confirms the deterministic results [Table 3]. In particular, the analysis showed that ranolazine was more cost-effective than SoC for all the simulations. The CEAC showed that the likelihood of ranolazine being cost-effective at a WTP threshold of 34,000€ was $100 \%$. Furthermore, in the alternative conservative WTP threshold of $€ 17,000$ the CEAC showed that the likelihood of ranolazine being cost-effective over SoC alone was $99.9 \%$ [Fig. 2].

\section{Discussion}

In the present study, an economic analysis was undertaken to compare, from a payer perspective, the ranolazine as add-on therapy to $\mathrm{SoC}$ relative with $\mathrm{SoC}$ alone during a 6-month period in patients with chronic stable angina in Greece. Ranolazine as add-on therapy to SoC was more expensive treatment regimen as it was found that the total 6-month treatment cost was higher by $€ 186$ compared to SoC alone. In terms of health outcomes, ranolazine was more effective as it reduces the severity and frequency of angina episodes in relation with SoC, resulting in higher QALYs gained. Hence, ranolazine was found to be a cost-effective treatment alternative, resulting in an ICER equal to $€ 4620$ per QALY gained well below the predetermined WTP threshold of $€ 34,000$ per QALY gained. Furthermore, the OWSA

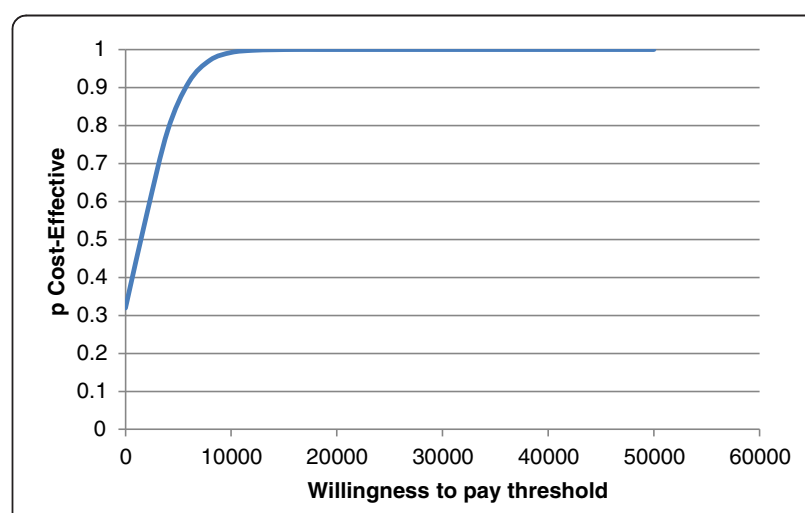

Fig. 2 Cost-effectiveness acceptability curve ranolazine plus SoC vs SoC alone 
revealed that ranolazine as add-on therapy to SoC remains a well cost- effective treatment across all scenarios.

Our findings are in the line with those presented in the previously conducted economic analyses comparing ranolazine as an add-on therapy with SoC alone [24, 36, 37]. More specifically, a cost-utility study conducted in Spain [24] showed that ranolazine is a highly efficient add-on therapy for the symptomatic treatment of chronic angina pectoris with an ICER of $€ 8455$ per QALY. Moreover, a study which was conducted in the USA [36] showed that ranolazine as add-on to $\mathrm{SoC}$ is a cost-effective treatment in patients with weekly or daily angina with an ICER of $\$ 32,682$ per QALY. Similar findings were obtained in a study conducted in Russia [37], which showed that the use of ranolazine in patients with $>3$ attacks of angina pectoris per week was clinically and economically substantiated. Additionally, a retrospective study which performed in USA [38] showed that adding ranolazine to the treatment regimen of patients was associated with lower rates of revascularization and lower total costs of care than SoC (ranolazine:\$13,961 vs nitrates :\$18,166 and beta blockers/ calcium channel blockers $: \$ 17,612)$.

The methodology adopted was based upon standard recommendations to conduct economic evaluation and extensive sensitivity and probabilistic analyses were conducted to fully explore uncertainty. However, potential limitations to this study should be considered. Firstly, in the present analysis it was assumed that the clinical data obtained from the published studies were applicable to the Greek health care setting. The use of this data may be questionable, however given the lack of local related data, this choice was the only source of relevant clinical data; one may argue that pivotal trials are almost universally used to build models for pricing and reimbursement decisions. Secondly, in the absence of local data, three local experts (cardiologists) with extensive clinical experience on the management of chronic stable angina were used to obtain local resource utilization and validate some of the assumptions considered in the model. This may raise concerns about the subjectivity of model inputs and leave space for challenging the study results. Nevertheless a series of sensitivity analyses indicated that our model and outcomes are valid, since the main results remained unchanged. Furthermore, the current analysis was conducted from the third party payer perspective and as such only direct costs were considered. However a more complete analysis from a broader (societal) perspective may also be worthwhile. True health care and patient direct and indirect costs are higher than those considered in the present analysis, and therefore the cost-utility of a new therapy may be more favorable from a societal perspective.
Finally, it should be noted that the results have to be considered in the strict Greek setting and on the basis of the present time resource and drug prices. If any of the underlying parameters change, so may the results and the conclusions of the analysis.

\section{Conclusions}

The present study suggests that ranolazine as add-on treatment provides greater health benefits and is more costly. However, the additional cost per QALY gained is well below the predetermined WTP threshold of $€ 34,000$ per QALY gained. Therefore, the present study indicates that ranolazine as add-on treatment can be considered a cost-effective alternative for the symptomatic treatment of patients with chronic stable angina in Greece.

\section{Additional file}

Additional file 1: Table S1. Drug acquisition cost considered in the model. (DOCX 79 kb)

\section{Competing interests}

The study was sponsored by Menarini Hellas. The study sponsor had no interference in the study design, data collection or writing of the manuscript. JP and CV have received speaker honoraria from Menarini Hellas and International. None of the other authors has any personal or financial conflict of interest.

\section{Authors' contributions}

G.K. and G.G adapted the model, conducted the analyses, interpreted the results and wrote the manuscript. N.M. supervised the study, contributed to results interpretation, and reviewed the manuscript. C.V., J.P. and J.K. were the medical experts provided local input data and reviewed the manuscript. All authors have read and approved the final manuscript.

\section{Acknowledgments}

Authors would like to thank Menarini Hellas that sponsored this study.

\section{Author details}

${ }^{1}$ The Stavros Niarchos Foundation-Collaborative Center for Clinical Epidemiology and Outcomes Research (CLEO), National and Kapodistrian University of Athens, School of Medicine, Athens, Greece. ${ }^{2} 1$ st Department of Cardiology, Athens University Hospital "Ippokrateio", Athens, Greece. ${ }^{3}$ Heart Failure Unit, Department of Cardiology, Athens University Hospital "Attikon", Athens, Greece. " Department of Cardiology, "Alexandra" Hospital, Athens, Greece. ${ }^{5}$ Department of Health Services Organization \& Management, National School of Public Health, Athens, Greece.

Received: 26 October 2015 Accepted: 10 December 2015

Published online: 18 December 2015

References

1. National Institute for Health and Care Excellence (NICE). Management of stable angina. In: NICE clinical guideline 126. 2012. https://www.nice.org.uk/ guidance/cg126/resources/stable-angina-management-35109453262021. Accessed 15, October 2015.

2. Fox K, Garcia MA, Ardissino D, Buszman P, Camici PG, Crea F, et al. Guidelines on the management of stable angina pectoris: executive summary: the task force on the management of stable angina pectoris of the European Society of Cardiology. Eur Heart J. 2006;27(11):1341-81.

3. Spertus JA, Jones P, McDonell M, Fan V, Fihn SD. Health status predicts long-term outcome in outpatients with coronary disease. Circulation. 2002;106(1):43-9. 
4. Arnold SV, Morrow DA, Lei Y, Cohen DJ, Mahoney EM, Braunwald E, et al. Economic impact of angina after an acute coronary syndrome: insights from the MERLIN-TIMI 36 trial. Circ Cardiovasc Qual Outcomes. 2009:2(4):344-53.

5. Stewart S, Murphy NF, Walker A, McGuire A, McMurray JJ. The current cost of angina pectoris to the National Health Service in the UK. Heart. 2003;89(8):848-53.

6. Kempf J, Buysman E, Brixner D. Health resource utilization and direct costs associated with angina for patients with coronary artery disease in a US managed care setting. Am Health Drug Benefits. 2011;4(6):353-61.

7. Reynolds MW, Frame D, Scheye R, Rose ME, George S, Watson JB, et al. A systematic review of the economic burden of chronic angina. Am J Manag Care. 2004;10(11 Suppl):S347-57.

8. Andrikopoulos G, Parissis J, Filippatos G, Nikolaou M, Pantos K, Voudris V, et al. Medical management of stable angina. Hellenic J Cardiol. 2014;55(4):272-80.

9. Gayet JL, Paganelli F, Cohen-Solal A. Update on the medical treatment of stable angina. Arch Cardiovasc Dis. 2011;104(10):536-44.

10. Tarkin JM, Kaski JC. Pharmacological treatment of chronic stable angina pectoris. Clin Med. 2013;13(1):63-70.

11. Boden WE, O'Rourke RA, Teo KK, Hartigan PM, Maron DJ, Kostuk WJ, et al. Optimal medical therapy with or without PCI for stable coronary disease. N Engl J Med. 2007:356(15):1503-16.

12. Keating GM. Ranolazine: a review of its use as add-on therapy in patients with chronic stable angina pectoris. Drugs. 2013;73(1):55-73.

13. Borer JS, Fox K, Jaillon P, Lerebours G, Ivabradine Investigators $G$. Antianginal and antiischemic effects of ivabradine, an I(f) inhibitor, in stable angina: a randomized, double-blind, multicentered, placebo-controlled trial. Circulation. 2003;107(6):817-23.

14. Chaitman BR, Ivleva AY, Ujda M, Lenis JH, Toth C, Stieber DM, et al. Antianginal efficacy of omapatrilat in patients with chronic angina pectoris. Am J Cardiol. 2005;95(11):1283-9.

15. Tardif JC, Ford I, Tendera M, Bourassa MG, Fox K, Investigators I. Efficacy of ivabradine, a new selective I(f) inhibitor, compared with atenolol in patients with chronic stable angina. Eur Heart J. 2005;26(23):2529-36.

16. Vicari RM, Chaitman B, Keefe D, Smith WB, Chrysant SG, Tonkon MJ, et al. Efficacy and safety of fasudil in patients with stable angina: a double-blind, placebo-controlled, phase 2 trial. J Am Coll Cardiol. 2005;46(10):1803-11.

17. Chaitman BR, Pepine CJ, Parker JO, Skopal J, Chumakova G, Kuch J, et al. Effects of ranolazine with atenolol, amlodipine, or diltiazem on exercise tolerance and angina frequency in patients with severe chronic angina: a randomized controlled trial. JAMA. 2004;291(3):309-16.

18. Chaitman BR, Skettino SL, Parker JO, Hanley P, Meluzin J, Kuch J, et al. Antiischemic effects and long-term survival during ranolazine monotherapy in patients with chronic severe angina. J Am Coll Cardiol. 2004;43(8):1375-82.

19. Song Y, Shryock JC, Wu L, Belardinelli L. Antagonism by ranolazine of the pro-arrhythmic effects of increasing late INa in guinea pig ventricular myocytes. J Cardiovasc Pharmacol. 2004;44(2):192-9.

20. Wu L, Shryock JC, Song Y, Li Y, Antzelevitch C, Belardinelli L. Antiarrhythmic effects of ranolazine in a guinea pig in vitro model of long-QT syndrome. J Pharmacol Exp Ther. 2004;310(2):599-605.

21. European Medicines Agency. Ranexa: summary of product characteristics. 2008. http://www.ema.europa.eu/docs/en_GB/document library/EPAR Product_Information/human/000805/WC500045937.pdf. Accessed 13 October 2014

22. Stone PH, Gratsiansky NA, Blokhin A, Huang IZ, Meng L, Investigators E. Antianginal efficacy of ranolazine when added to treatment with amlodipine: the ERICA (Efficacy of Ranolazine in Chronic Angina) trial. J Am Coll Cardiol. 2006:48(3):566-75.

23. Morrow DA, Scirica BM, Karwatowska-Prokopczuk E, Murphy SA, Budaj A, Varshavsky S, et al. Effects of ranolazine on recurrent cardiovascular events in patients with non-ST-elevation acute coronary syndromes: the MERLINTIMI 36 randomized trial. JAMA. 2007;297(16):1775-83.

24. Hidalgo-Vega A, Ramos-Goni JM, Villoro R. Cost-utility of ranolazine for the symptomatic treatment of patients with chronic angina pectoris in Spain. Eur J Health Econ. 2013;15(9):917-25.

25. Arnold SV, Morrow DA, Wang K, Lei Y, Mahoney EM, Scirica BM, et al. Effects of ranolazine on disease-specific health status and quality of life among patients with acute coronary syndromes: results from the MERLIN-TIMI 36 randomized trial. Circ Cardiovasc Qual Outcomes. 2008;1(2):107-15.
26. Etemad LR, McCollam PL. Total first-year costs of acute coronary syndrome in a managed care setting. J Manag Care Pharm. 2005;11(4):300-6.

27. Longworth $L$, Buxton MJ, Sculpher M, Smith DH. Estimating utility data from clinical indicators for patients with stable angina. Eur J Health Econ. 2005;6(4):347-53.

28. Karwatowska-Prokopczuk E, Wang W, Cheng ML, Zeng D, Schwartz PJ, Belardinelli $\mathrm{L}$. The risk of sudden cardiac death in patients with non-ST elevation acute coronary syndrome and prolonged QTc interval: effect of ranolazine. Europace. 2013;15(3):429-36.

29. Spertus JA, Winder JA, Dewhurst TA, Deyo RA, Prodzinski J, McDonell M, et al. Development and evaluation of the Seattle angina questionnaire: a new functional status measure for coronary artery disease. J Am Coll Cardiol. 1995:25(2):333-41.

30. Greek Ministry of Health. Drug price bulletin 2014. 2014. http://www.moh. gov.gr/articles/times-farmakwn/deltia-timwn. Accessed 8 February 2015.

31. Greek Ministry of Health. Diagnostic related groups FEK 9468/27-3-12. Athens. http://www.moh.gov.gr/articles/health/domes-kai-draseis-gia-thnygeia/kwdikopoihseis/709-kleista-enopoihmena-noshlia-1. Accessed 8 February 2015.

32. WHO. Choosing interventions that are cost effective (WHO-CHOICE): costeffectiveness thresholds. 2013. http://www.who.int/choice/en/. Accessed 28 February 2015.

33. International Monetary Fund. World economic outlook database. 2013. http://www.imf.org/external/pubs/ft/weo/2012/02/weodata/index.aspx. Accessed 15 February 2015.

34. Briggs $A$, Claxton $K$, Sculpher M. Decision modelling for health economic evaluation. In: Gray A, Briggs A, editors. Handbooks in health economic evaluation. 2006.

35. Barber JA, Thompson SG. Analysis of cost data in randomized trials: an application of the non-parametric bootstrap. Stat Med. 2000;19(23):3219-36.

36. Kohn CG, Parker MW, Limone BL, Coleman Cl. Cost-effectiveness of ranolazine added to standard-of-care treatment in patients with chronic stable angina pectoris. Am J Cardiol. 2014:113(8):1306-11.

37. Gorokhova SG. ea. Cost-effectiveeness of ranolazlne for the treatment of anglna Pectoris in Russla. Value Health. 2014;17:A323-686.

38. Phelps CE, Buysman EK, Gomez RG. Costs and clinical outcomes associated with use of ranolazine for treatment of angina. Clin Ther. 2012;34(6):1395-407.e4.

\section{Submit your next manuscript to BioMed Central and we will help you at every step:}

- We accept pre-submission inquiries

- Our selector tool helps you to find the most relevant journal

- We provide round the clock customer support

- Convenient online submission

- Thorough peer review

- Inclusion in PubMed and all major indexing services

- Maximum visibility for your research

Submit your manuscript at www.biomedcentral.com/submit 